\title{
Unsteady heat and mass transfer in gels, used as media for immobilizing micro bio-objects
}

\author{
Boris Pokusaev ${ }^{1, *}$, Andrey Vyazmin ${ }^{1,2}$, Sergey Karlov ${ }^{1}$, Dmitry Nekrasov ${ }^{1}$ and \\ Dmitry Skladnev ${ }^{3}$ \\ ${ }^{1}$ Moscow Polytechnical University, Department of Chemical Engineering, 105066, Moscow, Russia \\ ${ }^{2}$ Scientific Research Institute of Rubber Industry, 141312, Sergiev Posad, Russia \\ ${ }^{3}$ Research Centre of Biotechnology RAS, 117312, Moscow, Russia
}

\begin{abstract}
The idea that gels can be used as a carrier during the formation of ordered bio-structured elements for medical additive technology seems quite promissing. For experimental study we used agarose based gels. Such gels are appropriate for additive technologies used for the creation of matrix of bioreactors by $3 \mathrm{D}$ printing. We investigated the formation kinetics of different density gels, and studyid the diffusion in the gels using optical method. The paper presents new data on the thermophysical properties of the formed agarose based gels.
\end{abstract}

\section{Introduction}

The idea of growing tissues and organs in vitro using stem cells is not new. However, in order to implement this idea, it is necessary to create special bioreactors which would be capable of maintaining the required temperature, $\mathrm{pH}$ level, osmotic pressure, supplying cells with nutrients and oxygen, and removing their metabolic products [1]. The use of the additive manufacturing method to create tissues from stem cells [2] seems to be very promising. Such works are already carried out in the area of 3D-bioprinting [3].

Currently gels are considered as high-potential structuring material for formation of bioreactors with immobilized cells. Gel is a dispersive system with liquid dispersing medium, and the dispersion phase makes up a spatial structured mesh due to intermolecular interaction. The properties of gels allow forming bioreactors of complex configurations based on layer-by-layer application of gels of various concentrations and compositions. Agarose and other gels are used in microbiology to grow microorganisms [4].

The properties of gels are known to depend on the composition of a gel-forming medium and preparation method. There are many publications relating to the studies on the methods of gel synthesis and investigation of their properties (see, for instance, [5]). However, many properties of gels are common in terms of the application technology. For instance, the most significant peculiarities are manifested in the mass transfer processes in gels due to their instability and anisotropy [6].

Agarose gel is a typical gel which is formed from the solution when the temperature is lowered. We have studied the following aspects: structure of links formed in the disperse

* Corresponding author: pokusaev2005@yandex.ru 
phase of gel, physical-chemical properties of gels, as well as technology of gelation for various uses [7]. Nevertheless, many properties of gels, defining the possibility of their use for the creation of bioreactors based on additive technology, require further study. Here the aim is to carry out investigations of the kinetic of agarose gel formation depending on the concentration, determination of diffusion, and thermophysical properties of gels.

\section{Additive method to form bioreactor}

Consider the creation of technologically simple method of producing artificial biomaterials with application of 3D bioprinting. The problem is that the cultures of living cells are sensitive to external and internal influences. Deviation of the growing conditions from the optimum parameters leads to an inhibition of metabolic activity. To ensure minimal cells damage during 3D bioprinting a specific approach is proposed in order to avoid changes in the concentrations of the growth factors, and to protect immobilized cells from excess contact with oxygen. The approach is named "gel in gel". It is based on technology, where the basis for the layering of portions of the gel containing immobilized cells is in a thicker protective very dilute agarose gel, prepared with the addition of optimal concentration of essential growth factors. The scheme of bioreactor formation is shown in Fig. 1.

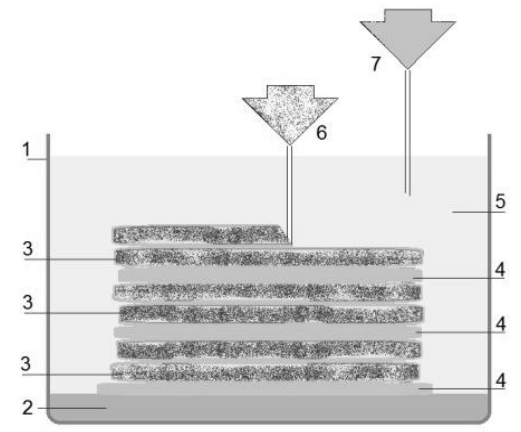

Fig. 1. The scheme of bioreactor created by using bioprinting method: 1 - the thermostatic cell, $2-$ underlying layer of sterile silica gel, 3 - layers of agarose gel with immobilized living cells, 4 - drain layers of sterile agarose gel, 5 - layer protection of low concentration agarose gel, 6 - device for the 3D layer formation with target cells, 7 - device for 3D-forming layers of sterile agarose gel.

It is proposed to use horizontal and vertical layers of gels of different density and composition. Gel layers containing immobilized cells alternate with the drainage layers, containing a gel with a low concentration of agarose. Drainage layers should be considered as an analogue of the natural microvascular networks in tissue.

Technological process of creating bioreactor is the following: gel base of sterile silica gel is applied to the bottom of the thermostatic cell by 3D bio-printing at a temperature of $37^{\circ} \mathrm{C}$. The working volume of thermostatic cell is filled with protective layer of agarose gel with low density ( $0.3 \%$ mass concentration of agsrose). Needle is injected into the cell for gel delivery for the formation of artificial biostructures. Agarose gel comprising a suspension of cells serves as a gel base at a temperature of $42^{\circ} \mathrm{C}$. Agarose gel comprising cells alternates with layers of sterile agarose gel of low concentration.

\section{Experimental equipments}

In order to study the kinetics of gel formation and diffusion mass transfer in the gels of different concentrations, we used a spectroscopy method. The illumination system is made of 35W Xenon lamp emitting light waves within the range of 185-2000 nm. Optical fibers 
$0.2 \mathrm{~mm}$ in diameter are used. The basic part of the experimental setup consists of spectrophotometer USB2000+VIS-NIR including Sony ILX511 2048-element detector. An optical cuvette of $10 \times 10 \mathrm{~mm}$ and $45 \mathrm{~mm}$ high contains the sample of gel and is installed in an air thermostat. A gel formation from the solution occurs when decreasing the temperature in the thermostat. The gel temperature range is chosen from 50 to $20^{\circ} \mathrm{C}$.

In order to measure the thermophysical properties of agarose gels, the experimental setup is equipped with a special heat flow sensor. Using a gradient heat flow sensor based on the Seebeck thermoelectric effect allows directing measurement of the amount of heat flow. The sensor is mounted on the side surface of a glass container of cylindrical shape with a diameter of $50 \mathrm{~mm}$ and $95 \mathrm{~mm}$ high. Thermocouples are fixed to the diameter cap to control the distribution of the temperature fields in the volume during gel formation. The working area is placed in a thermostat, where a constant temperature of $20^{\circ} \mathrm{C}$ is maintained.

In experiments we used «Chemapol» agarose. The gels are obtained by mixing agarose with distilled water. Agarose gels with $0.6-1.5 \%$ of agarose are used in the experiments.

\section{Results and discussion}

Figure 2 shows the experimental data on the change of wavelength, which coincides with the maximum of the light transmission spectrum, depending on the temperatures and the concentrations of agarose equal to $0.8 \%$ and $1.5 \%$. Along with the decrease of the temperature, the wavelengths suddenly increase at high temperatures, and then again take new constant values at low temperatures. This is caused by the increase of dispersion and light absorption of the selected wavelength when non-uniformities are less than a wavelength that is caused due to the change of the structure of the transmission medium.

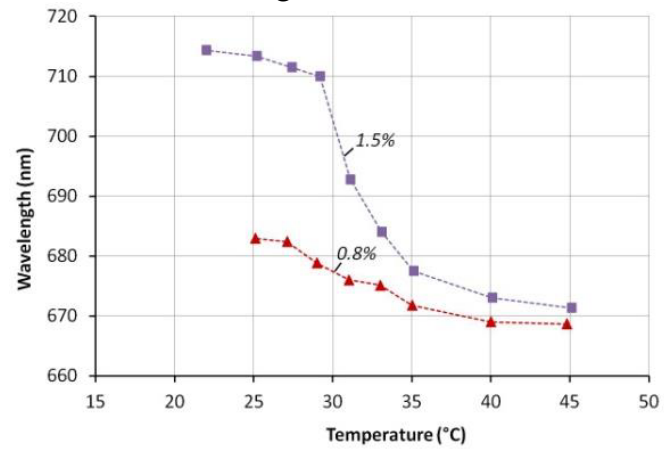

Fig. 2. The change in wavelength corresponding to the maximum of the spectrum of the transmitted light, depending on the temperature in the process of agarose gels formation

The dependencies shown in Fig. 2 are not equidistant for various agarose concentrations. This means that at high temperatures the solution shows weak dependency on the concentration. In the gel state, the structure of microdisperse medium significantly depends on the concentration.

Based on experimental data obtained by solving the inverse heat conduction problem, the average thermophysical coefficients for agarose gels of different concentrations within the temperature range of their formation are calculated. A mathematical model of nonstationary cooling of 1D cylindrical sample infinite in length with constant thermophysical properties (heat capacity and thermal conductivity) is considered for calculations.

To solve the inverse heat conduction problem, we used the extreme formulation and the principle of iterative regularization. The direct heat conduction problem is solved using an implicit difference scheme. The minimization problem is solved by the method of coordinate-wise descent. 
Thermal conductivity coefficients and bulk heat capacities averaged over the gel formation time for gels with different concentrations are obtained. Their values are given in Table 1 . The results show that within the experimental accuracy the heat capacity of gels of different concentrations is constant. Under the same conditions, the average thermal conductivity of gels decreases with increasing concentration of agarose.

Table 1. Averaged thermophysical properties of gels for different concentrations of agarose

\begin{tabular}{|c|c|c|}
\hline $\begin{array}{c}\text { Mass concentration } \\
\text { of agarose, } \%\end{array}$ & $\begin{array}{c}\text { Average thermal conductivity of } \\
\text { the gel, } \mathbf{W} /(\mathbf{m} \cdot \mathbf{K})\end{array}$ & $\begin{array}{c}\text { Average heat capacity of the gel, } \\
\mathbf{~} \mathbf{J}\left(\mathbf{m}^{\mathbf{3}} \cdot \mathbf{K}\right)\end{array}$ \\
\hline 0.6 & 0.538 & 2129.7 \\
\hline 1.0 & 0.501 & 2121.8 \\
\hline
\end{tabular}

The diffusion coefficients were determined experimentally by the method of moving boundaries in agarose gels with mass concentrations from 0.6 to $1.0 \%$ depending on the concentration of Peptone based LB bullion. The main results are shown on Fig. 3. Mass concentrations of the bullion ranged from 2.5 to $10 \%$. This is bullion with agarose gel standard medium for cultivation of microorganisms. It is established that the diffusion coefficients have an order of magnitude less than that for diffusion in liquids.

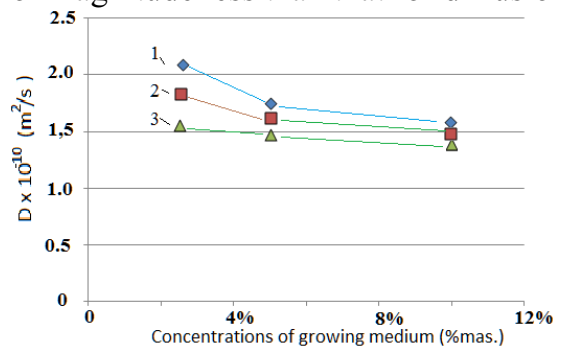

Fig. 3. The dependence of the diffusion coefficient of gel on concentration for nutrient bouillon. Notation: 1 -agarose gel concentration $0.6 \% ; 2-0.8 \% ; 3-1.0 \%$.

The obtained data allow evaluating just qualitatively the dependence of diffusion coefficients on the concentration of agarose gel and nourishing medium. With the growth of both concentrations, the diffusion coefficients slightly decrease.

This work was financially supported by the Russian Science Foundation (project No. 15-19-00177).

\section{References}

1. C.A.V. Rodrigues, T.G. Fernandes, M.M. Diogo, C.L. da Silva, J.M.S. Cabral, Biotech. Adv. 29, 815 (2011)

2. K. Hölzll, Sh. Lin, L. Tytgat, S. Van Vlierberghe, L. Gu, A. Ovsianikov, Biofabr. 8, 032002 (2016)

3. M.Y. Wang, J.K. He, Y.X. Liu, M. Li, D. Li, Zh. Jin, Int. J. Bioprint. 1, 15 (2015)

4. A.P. Hitchens, M.C. Leikind, J. Bacter. 37, 485 (1939)

5. R.G. Weiss, P. Terech, Molecular Gels: Materials with self-assembled fibrillar networks. (Springer Science \& Business Media, 2006)

6. B. Amsden, Macromolec. 31, 8382 (1998)

7. G.A. Santos, A manual for the processing of agar from Gracilaria (ASEAN Regional Small-Scale Coastal Fisheries Development Project, Manila, 1990) 